\title{
Author Index Volume 14
}

N. Ahmed see Ohkubo, S.

A. Y. Al Muzaffar \& K. A. Alshare

Determinants of Knowledge

Sharing in Virtual Network

Communities in Organizational

Context

D. G. Alemneh see Zavalina, O. L.

K. A. Alshare see Al Muzaffar, A. Y.

F. Barachini The Role of Power and Jealousy in Cooperation - A Framework for Artificial Agents

D. A. D. Bedford Enhancing Access to Knowledge Management Literature - A Proposal for Domain-Based Classification Scheme and Thesaurus

D. A. D. Bedford The Knowledge Management Information Landscape - Awareness, Access, Use and Value of Sources

I. Bhalla see Chakraverty, S.

O. Boussaid see Madani, A.

S. Burnett see Cleverley, P. H.

S. Chakraverty, S. Sharma \& I.

Bhalla Emotion-Location

Mapping and Analysis Using

Twitter

S.-C. Chang, C.-C. Sun, L.-Y. Pan \& M.-Y. Wang An Extended TAM to Explore Behavioural Intention of Consumers to Use M-Commerce

S. Chaveesuk see Vongjaturapat, S.

Q. Chen see Zhang, R.

C. W. Chong see Samadi, B.

N. Chotikakamthorn see

Vongjaturapat, S.
2 (2015) 1550014

2 (2015) 1550015

2 (2015) 1550003

1 (2015) 1550005

2 (2015) 1550003

1 (2015) 1550009

1 (2015) 1550006

2 (2015) 1550002

3 (2015) 1550022

3 (2015) 1550019

1 (2015) 1550007

3 (2015) 1550022

3 (2015) 1550023

4 (2015) 1550032

4 (2015) 1550034

3 (2015) 1550023
P. H. Cleverley \& S. Burnett Creating Sparks: Comparing

Search Results Using

Discriminatory Search Term

Word Co-Occurrence to Facilitate

Serendipity in the Enterprise

R. L. de Carvalho see de Mello, F. L.

F. L. de Mello \& R. L. de Carvalho Knowledge Geometry

D. Delen see Oztekin, A.

J. El-Den see Zhang, R.

S.-K. Goh \& P.-Y. Nee Pseudo Knowledge Sharing: The Influence of Trust and Guanxi Orientation

N. K. Hammah \& R. Ibrahim Workflow Complexities of Fit Criteria on Strategy Applications and Structure Adaptations

S. V. Harlan see Ohkubo, S.

S. Hawamdeh see Horne, S. B.

S. B. Horne \& S. Hawamdeh Factors Impacting the Implementation of Enterprise Content Management Systems

I. Hussain, A. Khan, A. Qurashi, Qurat-ul-Ain \& A. Shabbir The Impact of Knowledge Sharing Enablers on Employees' Performance: An Empirical Study on READ Foundation in Azad Jammu \& Kashmir

C. D. Idio see Majid, S.

Md. S. Islam, Md. A. K. Siddike,

S. Nowrin \& S. Naznin Usage and Applications of Knowledge Management for Improving Library and Information Services in Bangladesh
3 (2015) 1550020

2 (2015) 1550017

1 (2015) 1550004

1 (2015) 1550007

4 (2015) 1550028

4 (2015) 1550028

2 (2015) 1550013

4 (2015) 1550032

3 (2015) 1550025

2 (2015) 1550017

2 (2015) 1550015

1 (2015) 1550008

1 (2015) 1550008

3 (2015) 1550026 
Z. A. Karim see Noordin, M. F.

A. Khan see Hussain, I.

M. B. Khan see Khan, M. O. N.

M. O. N. Khan, S. ur Rahman \&

M. B. Khan The Synergistic

Effects of Knowledge Sharing and

Learning on Organisational

Learning: Demonstrating

Organisational Performance

N. K. Khilji \& S. A. Roberts $A n$

Exploratory Study of Knowledge

Management for Enhanced

Efficiency and Effectiveness: The

Transformation of the Planning

System in the UK Local

Government

P. Kizhakkethil see Zavalina, O. L.

C. C. Lee Identifying Knowledge

Gap to Meet Client Project

Requirements in Refurbishment

Projects

F. Liu Toward Wisdom: A

Hierarchical Wisdom Ontology

Based on Chinese Classics

A. Madani, O. Boussaid \& D. E.

Zegour New Information in

Trending Topics of Tweets by

Labelled Clusters

G. Maiga \& P. L. Mutuwa $A n$ Integrating Model of Knowledge Management for Improved

Pediatric Healthcare Practice

S. Majid, C. D. Idio, L. Shuang \&

Z. Wen Preferences and

Motivating Factors for Knowledge

Sharing by Students

M. N. Milhem see Radwan, S. F.

P. L. Mutuwa see Maiga, G.

S. Naznin see Islam, Md. S.

P.-Y. Nee see Goh, S.-K.

M. F. Noordin \& Z. A. Karim Modeling the Relationship between

Human Intelligence, Knowledge

Management Practices, and

Innovation Performance $\begin{array}{lll}1 & (2015) & 1550012 \\ 3 & (2015) & 1550020 \\ 3 & (2015) & 1550021\end{array}$

3 (2015) 1550021

1 (2015) 1550011

1 (2015) 1550005

2 (2015) 1550016

$4(2015) 1550027$

3 (2015) 1550019

2 (2015) 1550001

1 (2015) 1550004

4 (2015) 1550029

2 (2015) 1550001

3 (2015) 1550026

3 (2015) 1550025

1 (2015) 1550012
S. Nowrin see Islam, Md. S.

3 (2015) 1550026

S. Ohkubo, S. V. Harlan, N. Ahmed

\& R. M. Salem Conceptualising a

New Knowledge Management

Logic Model for Global Health: A Case-Study Approach

A. A. Olajide Social Media Platform as a Knowledge Management

Tool: Nigerian Librarians

Experience

A. Oztekin, D. Delen, H. Zaim,

A. Turkyilmaz \& S. Zaim The

Influence of Knowledge

Management on Financial and

Non-Financial Performance

L.-Y. Pan see Chang, S.-C.

S. Peterson \& L. Steelman Repatriate Knowledge Sharing Environment: Scale Development and Validation

M. E. Phillips see Zavalina, O. L.

A. Qurashi see Hussain, I.

Qurat-ul-Ain, see Hussain, I.

S. F. Radwan \& M. N. Milhem

Knowledge Management Towards

Connecting Minds - A Proposed

Concept for Expo 2020

S. A. Roberts see Khilji, N. K.

R. M. Salem see Ohkubo, S.

Samadi, B., C. W. Chong \& W. F. W. Yusoff The Influence of Trust on

Knowledge Sharing Behaviour

Among Multigenerational

Employees

U. Schmitt Quo Vadis, Knowledge Management: A Regeneration or a Revolution in the Making?

A. Shabir see Hussain, I.

S. Sharma see Chakraverty, S.

A. Shokri see Taghizadeh, H.

L. Shuang see Majid, S.

Md. A. K. Siddike see Islam, Md. S.

D. Soylu see Yilmaz, B.

L. Steelman see Peterson, S.

C.-C. Sun see Chang, S.-C.
$4(2015) 1550034$

$2(2015) 1550015$

3 (2015) 1550018

2 (2015) 1550013

2 (2015) 1550014

4 (2015) 1550031

1 (2015) 1550005

3 (2015) 1550020

3 (2015) 1550020

4 (2015) 1550029

1 (2015) 1550011

2 (2015) 1550015

4 (2015) 1550030

3 (2015) 1550020

3 (2015) 1550022

3 (2015) 1550024

1 (2015) 1550004

3 (2015) 1550026

1 (2015) 1550010

4 (2015) 1550031

2 (2015) 1550014 
H. Taghizadeh \& A. Shokri Relationship Among the Dimensions of Knowledge Management from the Viewpoint of Social Capital Based on Interpretive Structural Modelling (A Case Study)

3 (2015) 1550024

H. Tarver see Zavalina, O. L.

S. Tongkhambanchong see Vongjaturapat, S.

A. Turkyilmaz see Oztekin, A.

S. ur Rahman see Khan, M. O. N.

S. Vongjaturapat, S. Chaveesuk,

N. Chotikakamthorn \&

S. Tongkhambanchong Analysis of Factor Influencing the Tablet Acceptance for Library

Information Services: A Combination of UTAUT and TTF Model

3 (2015) 1550023

M.-Y. Wang see Chang, S.-C.

1 (2015) 1550005

3 (2015) 1550023

2 (2015) 1550013

3 (2015) 1550021
Z. Wen see Majid, S.

1 (2015) 1550004

D. Williams Nuts and Bolts of a Knowledge Management System $4(2015) 1550034$

B. Yilmaz \& D. Soylu SelfCensorship in Social Media: A Case Study of Hacettepe University

1 (2015) 1550010

W. F. W. Yusoff see Samadi, B.

H. Zaim see Oztekin, A.

4 (2015) 1550034

2 (2015) 1550013

S. Zaim see Oztekin, A.

2 (2015) 1550013

O. L. Zavalina, P. Kizhakkethil, D. G. Alemneh, M. E. Phillips \& H. Tarver Building a Framework of Metadata Change to Support Knowledge Management

1 (2015) 1550005

D. E. Zegour see Madani, A.

R. Zhang, J. El-Den \& Q. Chen Knowledge Sharing in Enterprise Business Simulative Games: An Empirical Analysis
3 (2015) 1550019

$4(2015) 1550032$ 